\title{
Experimental and Numerical Investigation of Heat Transfer Processes in Rocket Engine Cooling Channels Operated with Cryogenic Hydrogen and Methane at Supercritical Conditions
}

\author{
By Jan HAEMISCH, ${ }^{1)}$ Dmitry Suslov,${ }^{1)}$ and Michael OsChWALD ${ }^{1)}$ \\ ${ }^{1)}$ German Aerospace Center, DLR Lampoldshausen Institute of Space Propulsion
}

\begin{abstract}
Hydrogen and Methane are two fluids that are either used or in discussion as propellants for upper and lower stage rocket engines. The conception of a regenerative cooling system is a crucial part in the design of a rocket engine and so is the detailed knowledge of the coolants behavior and the heat transfer capabilities. Hydrogen is a very efficient and well known cooling fluid whereas the properties of methane as a cooling fluid are intensively investigated nowadays.

Experiments were performed with a subscale combustion chamber that is divided into four sectors around the circumference each containing rectangular cooling channels with different aspect ratios. Cryogenic hydrogen and liquid methane were used as cooling fluids. These experiments provide a broad data basis that is used for the validation of CFD simulations. The simulations are capable of predicting wall temperatures for high pressure conditions. Thermal stratification effects that are known to limit cooling properties in high aspect ratio cooling channels arise for both fluids, but the effects are much stronger for hydrogen compared to methane. However in the vicinity to the critical point, when it comes to heat transfer deterioration, the simulations show large deviations to the experimental values.
\end{abstract}

Key Words: Methane, Hydrogen, Thermal Stratification, Heat Transfer Deterioration, Supercritical, Regenerative Cooling, HTD, HARCC

\section{Introduction}

In recent years methane is in discussion as a replacement for hydrogen as a fuel for liquid rocket engines. It has a much higher density, compared to hydrogen and can therefore be stored in smaller, lighter tanks. Additionally insulation can be saved due to a higher boiling point. Major drawback is a reduction of the specific impuls. Nevertheless multiple $\mathrm{LOX} / \mathrm{CH}_{4}$ or LOX/LNG engines are currently under development. ${ }^{2,4,8)}$

One of the major concerns regarding methane as a fuel are the cooling capabilities in regenerative cooling channels. In the 1960s high effort was taken to investigate and optimize cooling channels for the regenerative cooling with hydrogen as coolant. ${ }^{17)}$ Cooling channels with a high aspect ratio enlarge the cooling channel area and lead, in combination with a high thermal conductivity of the wall material, to a significant improvement in both heat transfer and pressure loss inside the cooling channels. ${ }^{23,24)}$ However Kacynski ${ }^{7)}$ postulated a thermal stratification in very high aspect ratio cooling channels due to a limited mixing within these channels. This stratification leads to hot fluid at the bottom and cold fluid in the upper part of the channel and thus limits the heat transfer capabilities. The impact of this effect is intensively studied both experimental ${ }^{25,25)}$ and numerically ${ }^{11,15,16)}$ with hydrogen as coolant.

Cooling with methane is mainly driven by the vicinity to the critical point. Figure 1 shows the inlet and outlet conditions for hydrogen and methane as a function of reduced pressure and reduced temperature. Hydrogen enters the cooling channel at supercritical pressure and temperature. In contrast to that, methane enters the cooling channel at supercritical pressure but subcritical temperature. It is then heated up in the cooling channels and crosses the widom-line that is the extension of the co-

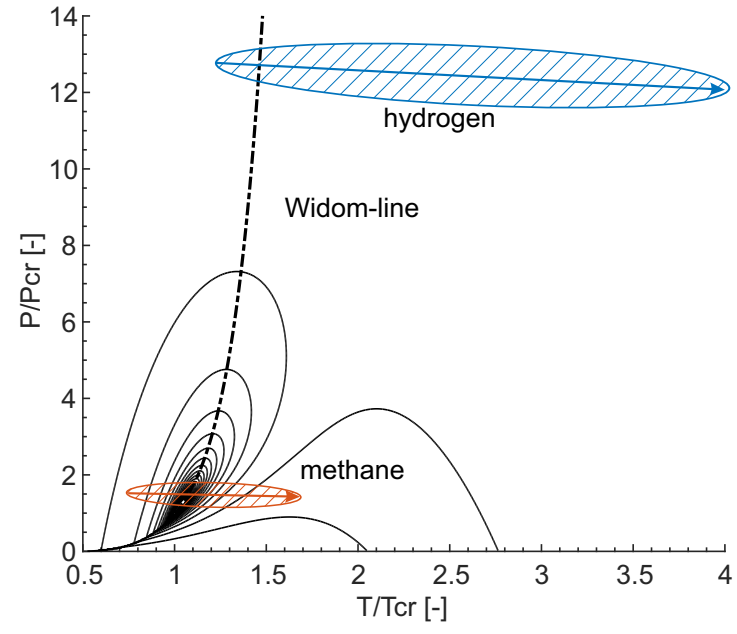

Fig. 1. Operational conditions for hydrogen and methane as a function of reduced pressure and reduced temperature.

existence line into the supercritical domain. The widom-line is defined as the line where the specific heat capacity at constant pressure $c_{P}$ exhibits a maximum value. ${ }^{3)}$ In that region the physical properties of a fluid change with small differences in temperature and pressure. In addition to a high heat flux this may lead to a separation of hot gas-like fluid close to the wall and cold liquid-like fluid in the bulk flow. The local reduction of heat transfer coefficient due to this effect is called heat transfer deterioration and is a major concern regarding methane as coolant. ${ }^{6,14,22)}$

To study both effects, thermal stratification and heat transfer deterioration, experiments were conducted with a subscale research combustion chamber. 
Table 1. Channel geometry.

\begin{tabular}{lllll}
\hline sector & S1 & S2 & S3 & S4 \\
\hline height $[\mathrm{mm}]$ & 2.0 & 2.8 & 30 & 9.2 \\
width [mm] & 1.2 & 0.8 & 0.3 & 0.5 \\
aspect ratio [-] & 1.7 & 3.5 & 30 & 9.2 \\
number of cooling channels $\left(\right.$ per $\left.90^{\circ}\right)$ & 25 & 29 & 38 & 34 \\
\hline
\end{tabular}

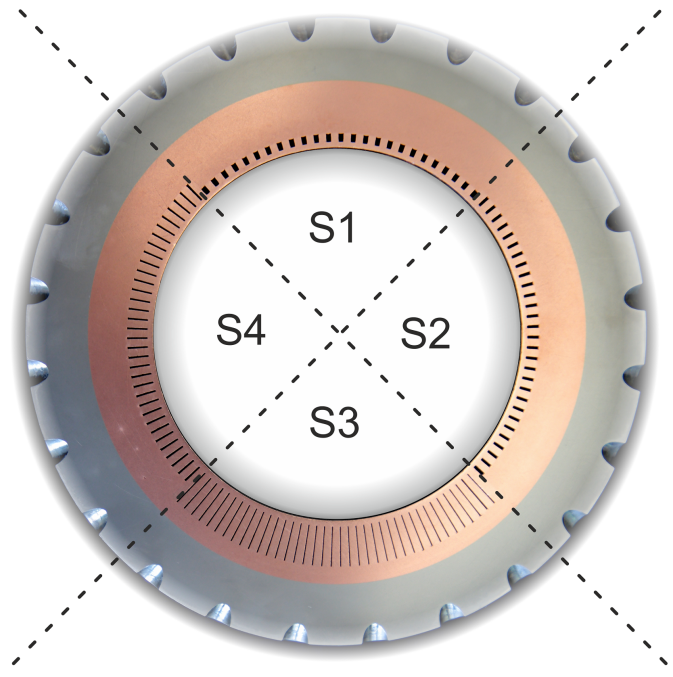

Fig. 2. Cut through the HARCC Segment

\section{Experimental Setup}

\subsection{The HARCC Segment}

To investigate the heat transfer for hydrogen and methane in cooling channels with various aspect ratios the HARCCSegment (High Aspect Ratio Cooling Channels) was developed. ${ }^{20)}$ The HARCC-Segment is a cylindrical combustion chamber segment that has an inner diameter of $D=80 \mathrm{~mm}$ and a length of $L=200 \mathrm{~mm}$. It is divided into four sectors around the circumference, each containing different cooling channel geometries. Those are rectangular cooling channels with the aspect ratios 1.7, 3.5, 9.2, and 30 (see figs 2, 3 and tab. 1). The cooling channels are wire cut and closed with electro deposit copper and nickel. Due to this manufacturing process the true geometry slightly differs from the ideal rectangular shape. Figure 3 shows a picture taken after the succesfull test campaign, when the segment was cut into slices. The edges are not precisely $90^{\circ}$, on the bottom of the cooling channels due to the wire diameter, the radius is $r \approx 0.15 \mathrm{~mm}$. The upper part of the channel is influenced by the electro deposit copper that slightly reduces the cooling channel area.

The HARCC Segment is part of the combustion chamber " $D$ "' that has an injector head with 42 coaxial injection elements. ${ }^{18)}$ Due to this large number, the heat flux around the combustion chamber circumference is assumed to be even. In addition to that a $200 \mathrm{~mm}$ long cylindrical Standard-Segment is placed between the injector head and the HARCC-Segment. Influences of the injector head or uncompleted combustion on the HARCC-Segment are therefore neglected. Figure 4 shows the distribution of cooling fluids inside the combustion chamber. The Standard-Segment is counter-flow cooled, the HARCCSegment is co-flow cooled. In that way, axial heat flux from one segment to the other is prevented. For the same reason the nozzle is counter-flow cooled with hydrogen, for the hydro-

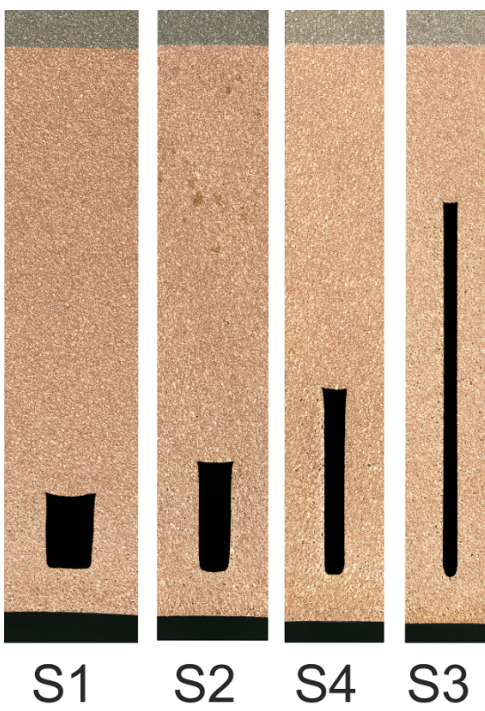

Fig. 3. Detailed picture that reveals the true geometrie of the four sectors.

gen test case but, due to the different fluid temperature co-flow cooled with water for the methane test case. For both test cases the fluid temperatures at the interfaces are similar and axial heat flux is prevented.

The mass flow that is used to cool the Standard-Segment is injected into the combustion chamber whereas the mass flow that is used to cool the HARCC-Segment is dumped. In that way the HARCC-Segment can be controlled independently from the remaining combustion chamber.

Orifices in the HARCC-Segment ensure that all sectors are fed with nearly the same mass flow.

\subsection{Measurement technique}

The combustion chamber is intensively equipped with measurement techniques. The mass flow for the Standard-Segment, the nozzle and each of the 4 sectors of the HARCC-Segment is measured with measurement turbines as well as pressure and temperature sensors inside the piping system. The inlet and outlet manifolds of each segment and sector are equipped with pressure and temperature sensors to estimate the average heat flux based on the calorimetric method. The calorimetric method calculates the average heat flux according eq. 1 with the enthalpy difference between inlet and outlet and the mass flow.

$$
\dot{q}_{w}=\frac{\dot{m}}{A}\left(h_{\text {out }}\left(P_{\text {out }}, T_{\text {out }}\right)-h_{\text {in }}\left(P_{\text {in }}, T_{\text {in }}\right)\right)
$$

The most important measures for the analysis of the heat transfer are 80 thermocouples, 20 per sector that are integrated into the combustion chamber wall between two cooling channels. These are integrated at four measurement positions at 5 different distances to the hot gas side. Table 2 summarize the positions and fig. 5 shows the distribution of thermocouples compared to the cooling channels in true dimensions.

The thermocouples are thermally insulated and springpressed into the drill holes. This method significantly improves the measurement signal. ${ }^{20,21)}$

These temperature values can be used to numerically recalculate the thermal field with an inverse temperature method. $5,6,9,13,20)$ The boundary conditions for this method are 


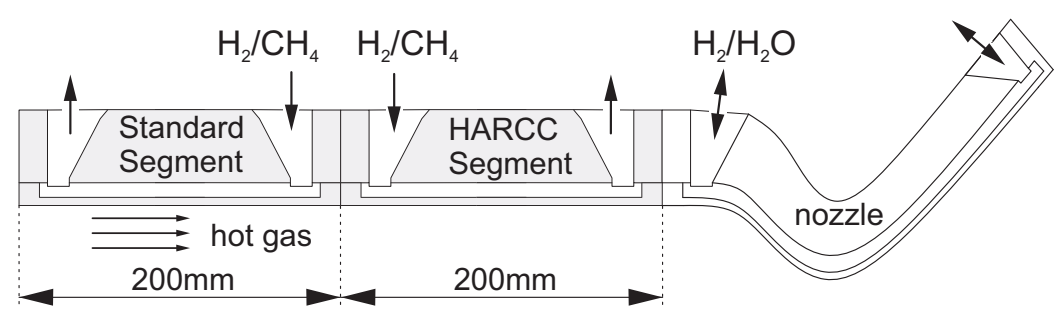

Fig. 4. Distribution of cooling fluids inside the combustion chamber (not to scale).

Table 2. Position of the thermocouples in the structure of the HARCCSegment

\begin{tabular}{llllll}
\hline axial position & $\mathrm{P} 1$ & $\mathrm{P} 1$ & $\mathrm{P} 3$ & $\mathrm{P} 4$ & \\
\hline distance from the inlet $[\mathrm{mm}]$ & 52 & 85 & 119 & 152 & \\
\hline radial position & $T_{1}$ & $T_{2}$ & $T_{3}$ & $T_{4}$ & $T_{5}$ \\
\hline distance from hot gas $[\mathrm{mm}]$ & 0.7 & 1.1 & 1.5 & 1.9 & 7.5 \\
\hline
\end{tabular}

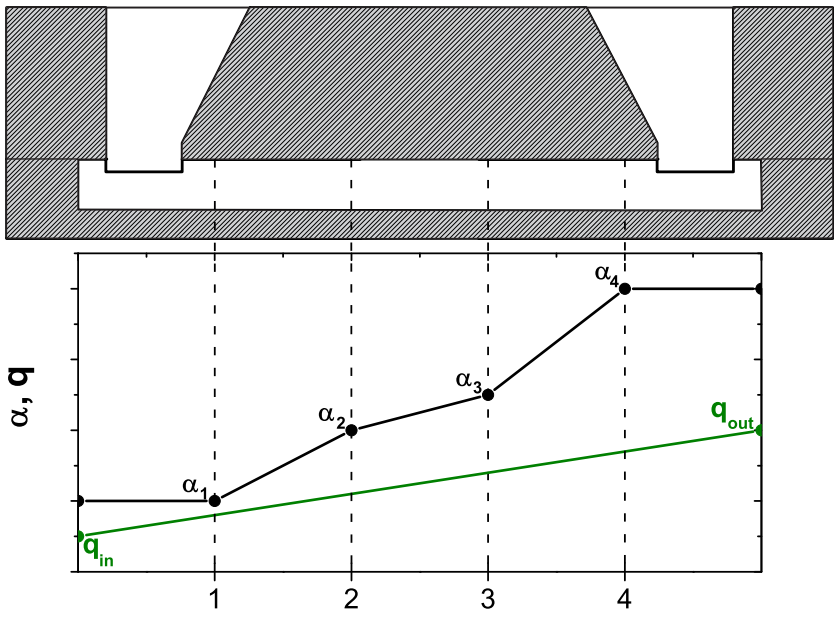

Fig. 6. Boundary conditions for the inverse method (not to scale)

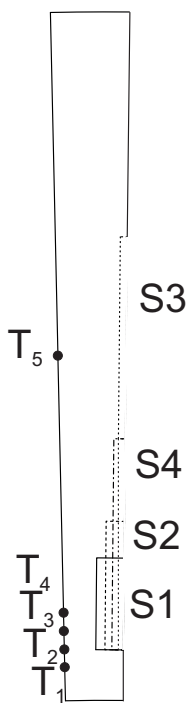

Fig. 5. Thermocouples and channel geometry in true dimension.

sketched in fig 6. The heat fluxes $q_{\text {in }}$ and $q_{\text {out }}$ as well as the heat transfer coefficients $\alpha_{1-4}$ are adjusted by an optimization algorithm until the measured and calculated temperature values collide. This method is described in more detail in. ${ }^{5,6,13)}$ The inverse method provides a linear heat flux distribution at the hot gas side that is used as a boundary condition for the numerical simulations. Comparison between inverse method and common calorimetric method shows satisfactory small deviations., ${ }^{5,6}$

\section{Numerical Setup}

The experimental results are used to validate numerical simulations performed with the commercial software ANSYS CFX 18.0. The boundary conditions are taken from the experiment and are summarized in tabs. 4 and 5. At the inlet the measured mass flow and temperature are used and at the outlet the static pressure that is measured in the outlet manifold. At the hot gas side two approaches are considered: 1 . constant heat flux calculated with common calorimetric method. 2. linear heat flux calculated with the inverse method. Both approaches were used with the ideal cooling channel geometry (tab. 1) and the second approach was used with the ideal and the real measured geometry (fig. 3).

Due to symmetry only half a channel is simulated. At the

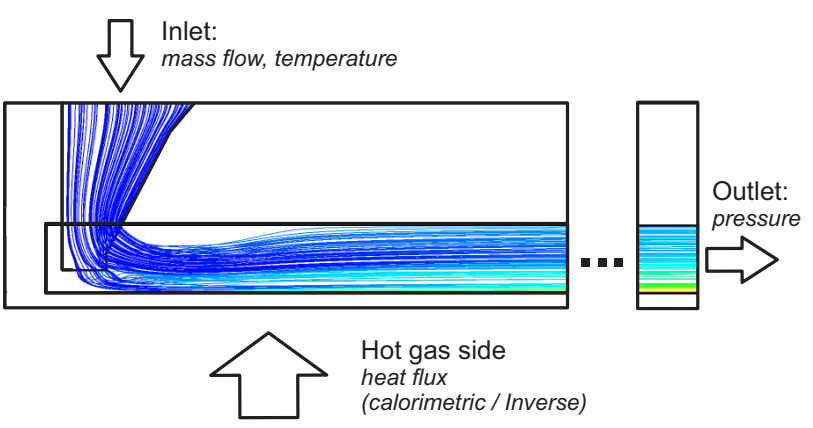

Fig. 7. Geometry and boundary conditions for the numerical setup.

inlet the flow is turned by $90^{\circ}$. The outlet manifold is not considered. Figure 7 shows an example of the geometry and the used boundary conditions.

As turbulence model the shear stress transport (SST) model is used. ${ }^{12)}$ The used mesh is a structured hexa-mesh that is refined towards the walls and ensures a $y^{+}$-value of $\approx 1$.

Before closing the cooling channels were highly polished. ${ }^{25)}$ The roughness of such a polished area was measured at the nickel part of a polished slice. The roughness was measured to $R_{a}=0.196 \mu m$. Equivalent sand grain roughness was calculated using equation 2. ${ }^{1)}$ This value is used for the numerical simulations.

$$
k_{s}=5.86 \cdot R_{a} \approx 1.1 \mu \mathrm{m}
$$

Thermal conductivity of the used copper alloy is: $\lambda=350 \frac{\mathrm{W}}{\mathrm{mK}}$.

The fluid properties are applied using real gas tables for hydrogen ${ }^{10)}$ and methane. ${ }^{19)}$ 
Table 3. Test cases to directly compare hydrogen and methane.

\begin{tabular}{lll}
\hline & hydrogen & methane \\
\hline$P_{c c}[$ bar $]$ & 49.1 & 50.2 \\
$R O F[-]$ & 3.9 & 2.0 \\
$F[k N]$ & 17.0 & 17.8 \\
$\dot{m}_{I N J}\left[\frac{\mathrm{kg}}{\mathrm{s}}\right]$ & 0.79 & 1.85 \\
$\dot{m}_{H A R C C}\left[\frac{\mathrm{kg}}{\mathrm{s}}\right]$ & 0.65 & 1.74 \\
$\frac{\dot{m}_{H A R C C}[-]}{\dot{m}_{I N J}}[0.82$ & 0.94 \\
$T_{i n}[K]$ & $\sim 65$ & $\sim 139$ \\
\hline
\end{tabular}

Table 4. Boundary conditions for the simulation with hydrogen

\begin{tabular}{lllll}
\hline & $\mathrm{S} 1$ & $\mathrm{~S} 2$ & $\mathrm{~S} 3$ & $\mathrm{~S} 4$ \\
\hline$\dot{m}[\mathrm{~g} / \mathrm{s} /$ channel $]$ & 7.5 & 5.5 & 4.1 & 4.4 \\
$T_{\text {in }}[\mathrm{K}]$ & 63.5 & 65.8 & 65.0 & 66.0 \\
$P_{\text {out }}[$ bar $]$ & 155.3 & 155.7 & 154.5 & 155.6 \\
$\dot{q}_{w}($ calo $)\left[\mathrm{MW} / \mathrm{m}^{2}\right]$ & 26.3 & 22.7 & 16.7 & 21.5 \\
$\dot{q}_{w, \text { in }}($ inverse $)\left[\mathrm{MW} / \mathrm{m}^{2}\right]$ & 20.1 & 21.5 & 17.0 & 23.0 \\
$\dot{q}_{w, \text { out }}($ inverse $)\left[\mathrm{MW} / \mathrm{m}^{2}\right]$ & 25.2 & 18.4 & 16.4 & 16.5 \\
\hline
\end{tabular}

\section{Results}

In this section the numerical results for the two test cases are shown and compared to the experimental results.

The test cases are chosen due to a similar thrust and a similar combustion chamber pressure of $P_{c c} \approx 50 \mathrm{bar}$. The test cases and boundary conditions are summarized in tabs. 3 - 5 .

The thrust depends on the mass flow and specific impulse.

$$
F=\dot{m} \cdot g \cdot I_{S P}
$$

For the same thrust this leads to:

$$
\dot{m}_{H 2, t o t} \cdot I_{S P, H 2}=\dot{m}_{C H 4, t o t} \cdot I_{S P, C H 4}
$$

The total mass flow is the sum of fuel mass flow $\dot{m}_{H 2}$ respectively $\dot{m}_{\mathrm{CH} 4}$ and oxidizer mass flow $\dot{m}_{\mathrm{O} 2}$. Both are linked with the mixture ratio $R O F$.

$$
R O F=\frac{\dot{m}_{O 2}}{\dot{m}_{H 2 / C H 4}}
$$

That leads to a methane mass flow of:

$$
\dot{m}_{C H 4}=\frac{I_{S P, H 2}}{I_{S P, C H 4}} \frac{1+R O F_{H 2}}{1+R O F_{C H 4}} \dot{m}_{H 2}
$$

Because the coolant mass flow is not equal to the mass flow that is fed into the combustion chamber, the ratio of coolant mass flow to injector mass flow has to be considered. In the analyzed test cases the ratio $\frac{\dot{m}_{H A R C C}}{\dot{m}_{I N J}}$ is 0.82 for the hydrogen test case and 0.94 for the methane test case. So the equivalent

Table 5. Boundary conditions for the simulation with methane.

\begin{tabular}{lllll}
\hline & $\mathrm{S} 1$ & $\mathrm{~S} 2$ & $\mathrm{~S} 3$ & $\mathrm{~S} 4$ \\
\hline$\dot{m}[\mathrm{~g} / \mathrm{s} /$ channel $]$ & 20.1 & 15.0 & 11.0 & 11.3 \\
$T_{\text {in }}[\mathrm{K}]$ & 138.6 & 139.5 & 139.1 & 139.8 \\
$P_{\text {out }}[$ bar $]$ & 78.1 & 77.8 & 78.0 & 78.6 \\
$\dot{q}_{w}($ calo $)\left[\mathrm{MW} / \mathrm{m}^{2}\right]$ & 14.5 & 13.3 & 11.4 & 16.0 \\
$\dot{q}_{w, \text { in }}($ inverse $)\left[\mathrm{MW} / \mathrm{m}^{2}\right]$ & 13.7 & 14.2 & 12.5 & 13.9 \\
$\dot{q}_{w, \text { out }}$ (inverse $)\left[\mathrm{MW} / \mathrm{m}^{2}\right]$ & 14.7 & 12.5 & 14.9 & 13.8 \\
\hline
\end{tabular}

coolant mass flow for the methane test case is relatively higher compared to the hydrogen test case.

\subsection{Hot gas side wall temperature}

Figure 8 shows the hot gas side wall temperature along the cooling channel length for all four sectors for hydrogen as coolant. At the entrance, due to the unheated part followed by the manifold that turns the flow $90^{\circ}$ (see fig. 7) the hot gas side wall temperature exhibits a strong decrease followed by a strong increase. This entrance effect influences the temperature until about $x \approx 0.05 \mathrm{~m}$ that is the first measurement position. After this point, the wall temperature increases monotonically with a nearly constant slope.

The magnitude of this slope rises with increasing aspect ratio. This effect can be seen for test cases with a constant (calorimetric) heat flux (red lines in fig. 8). The higher the aspect ratio, the higher the temperature increase between $x=0.05 \mathrm{~m}$ and $x=0.2 \mathrm{~m}$. This outcome is due to thermal stratification that is also discussed in more detail in sec. 4.3. However when compared to the test cases with a linear heat flux and especially the experimental data, the impact of thermal stratification on hot gas side wall temperature is small.

The comparison of ideal rectangular (blue line) to real measured geometry (green line) shows very small deviations, thus the influence of the corner as well as the upper part can be neglected. Especially for cooling channel S3 that exhibits a round lower part (see fig. 3) has perfect alignment with the ideal shaped result. So one can conclude that the side part of the cooling channels have a way higher impact on heat transfer compared to the top and the bottom walls.

The same trends can be seen for methane as coolant. The hot gas side wall temperature for these test cases are shown in fig. 9. The entrance effect is less pronounced. The impact of thermal stratification is dominated by other effects. The temperature increase is higher for lower aspect ratios. The results for the ideal and the real geometry are equal and the results with a linear heat flux at the hot gas side boundary show a very good agreement with the experimental results. Only for S1 (AR:1.7) the simulation overpredicts the experiment. Note that the scale in fig. 9 was adjusted for this case.

Comparing hydrogen and methane, the temperature gradients are higher for methane. This trend is even more distinct the lower the aspect ratio of the cooling channel is. The lower the aspect ratio the steeper the gradient. For sector 3 that has the highest aspect ratio, the gradients are very similar. Thus one can conclude that thermal stratification is of minor relevance for methane and is dominated by other effects (at least when regarding hot gas side wall temperature).

In summary the test cases with methane exhibit higher temperatures for all sectors compared to hydrogen.

\subsection{Structural temperatures}

Figures 10 and 11 show a direct comparison of experimentally measured and simulated temperature values inside the structure between two cooling channels. Simulated was the ideal rectangular shape with linear heat flux boundary condition. The overall comparison is very good for both coolants and all aspect ratios (with the exception of $\mathrm{S} 1$ cooled with methane). Higher deviations are for the low aspect ratio channels S1 \& S2 cooled with hydrogen for the temperature values far away from 


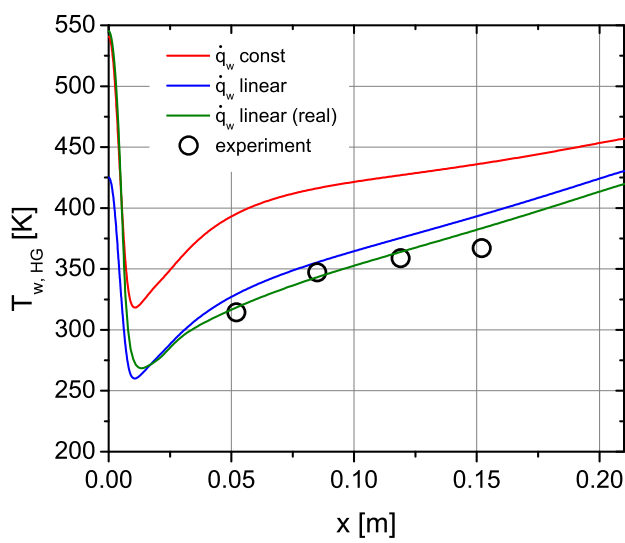

(a) S1 (AR:1,7)

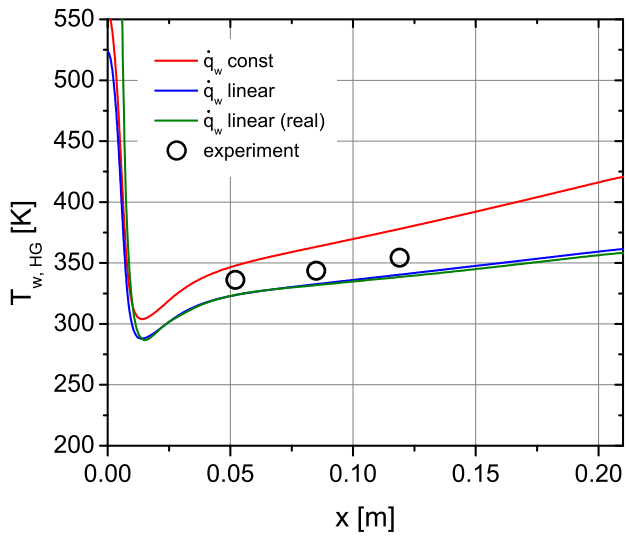

(b) S2 (AR:3,5)

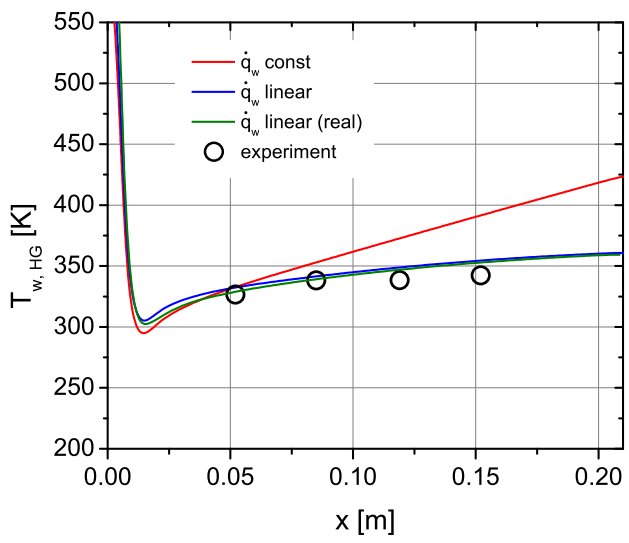

(c) S4 (AR:9,2)

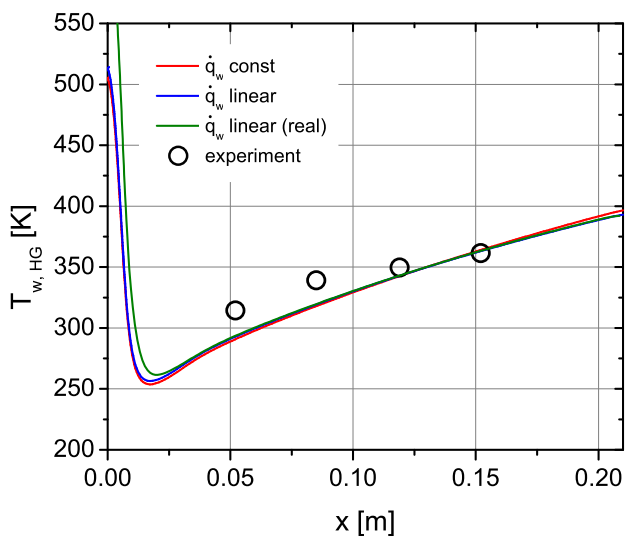

(d) S3 (AR:30)

Fig. 8. Hot gas side wall temperatures for cooling with hydrogen. Simulations with the results for the kalorimetric and inverse mathod as well as for the ideal and real geometry.

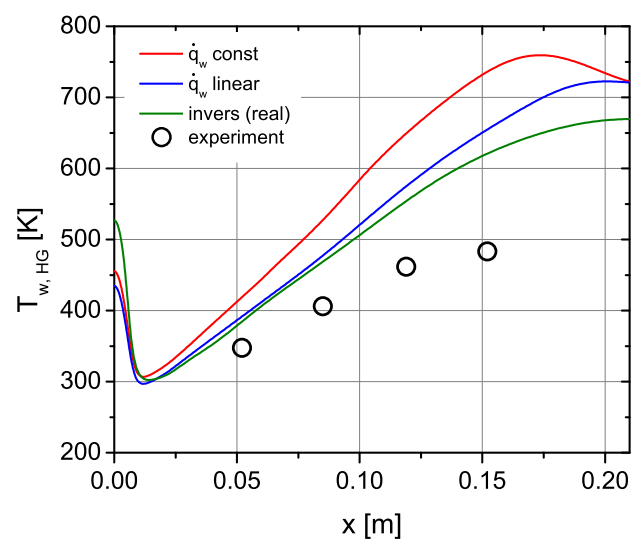

(a) $\mathrm{S} 1(\mathrm{AR}: 1,7)$

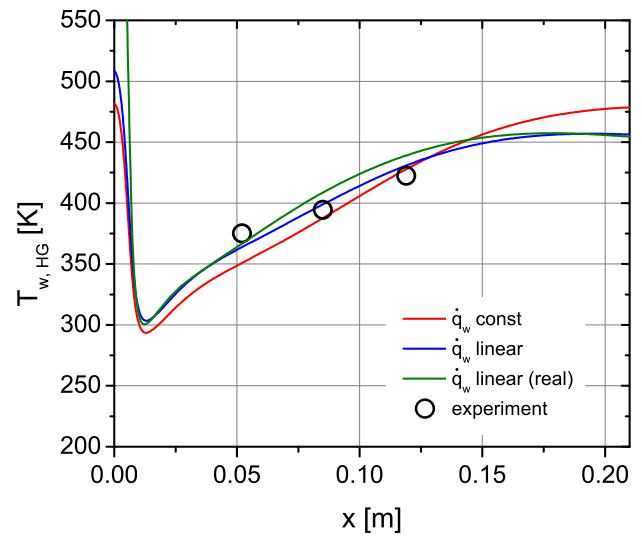

(b) S2 (AR:3,5)

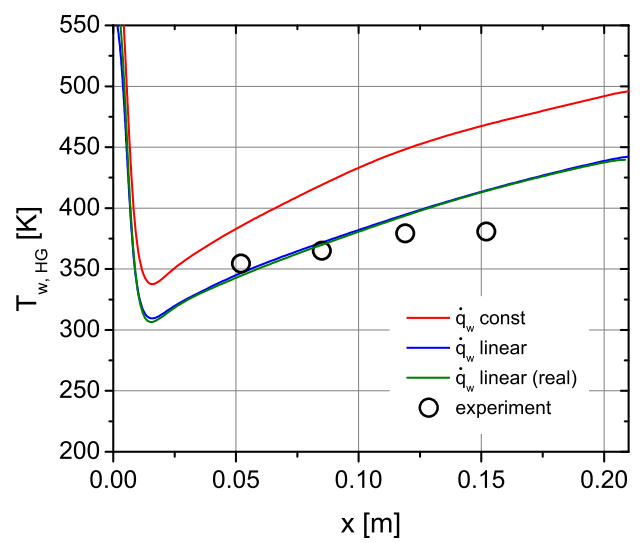

(c) S4 (AR:9,2)

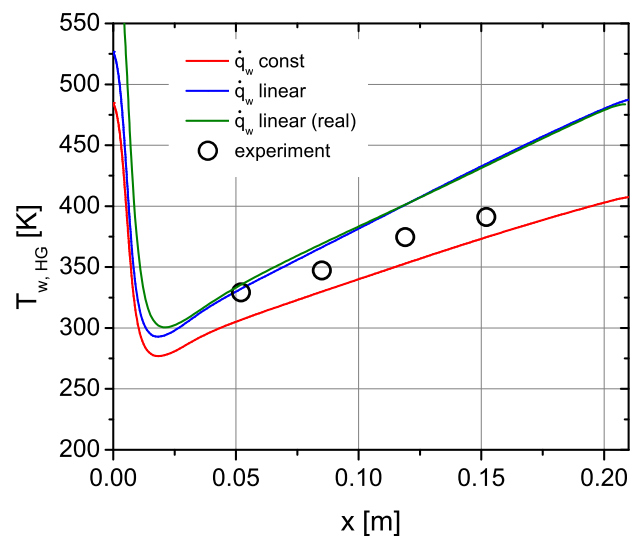

(d) S3 (AR:30)

Fig. 9. Hot gas side wall temperatures for cooling with methane. Simulations with the results for the kalorimetric and inverse mathod as well as for the ideal and real geometry. 


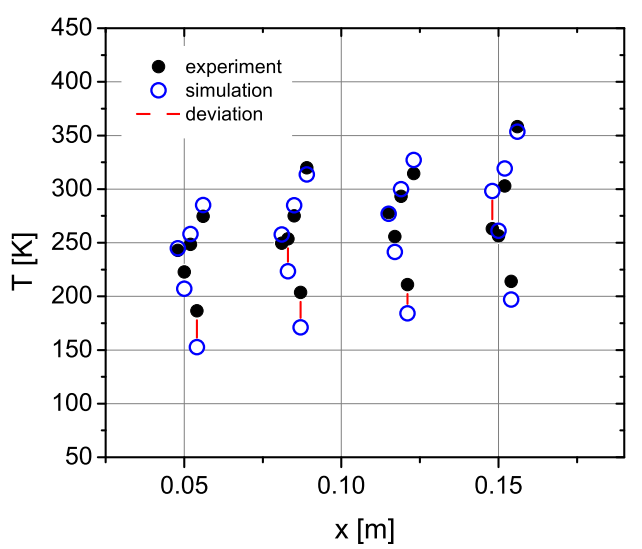

(a) $\mathrm{S} 1(\mathrm{AR}: 1,7)$

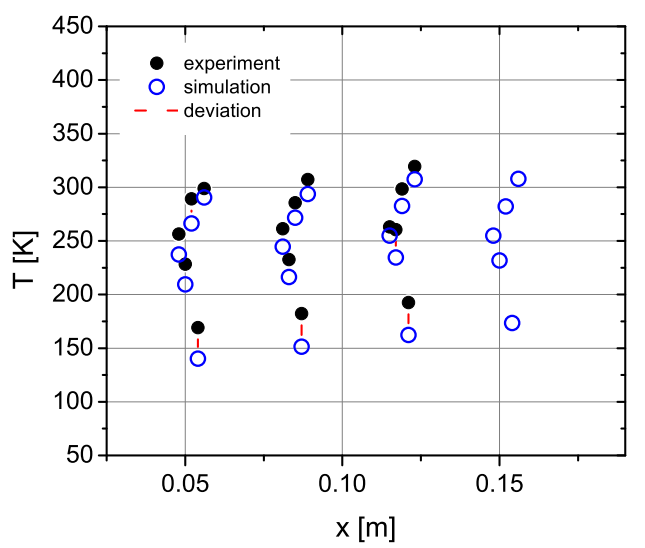

(b) $\mathrm{S} 2(\mathrm{AR}: 3,5)$

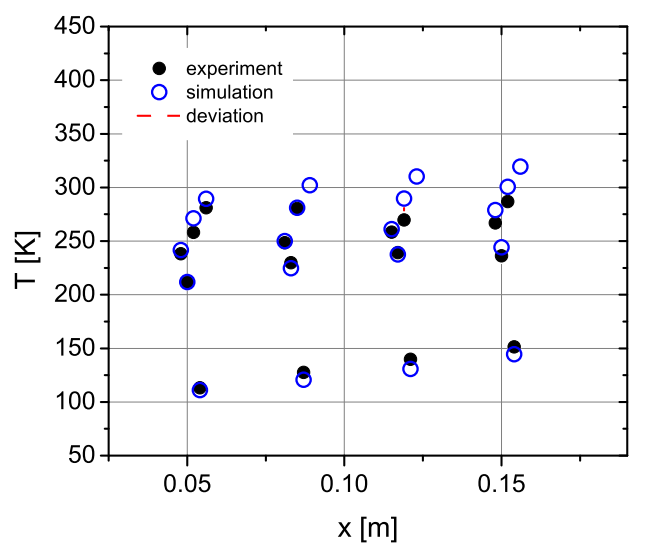

(c) $\mathrm{S} 4(\mathrm{AR}: 9,2)$

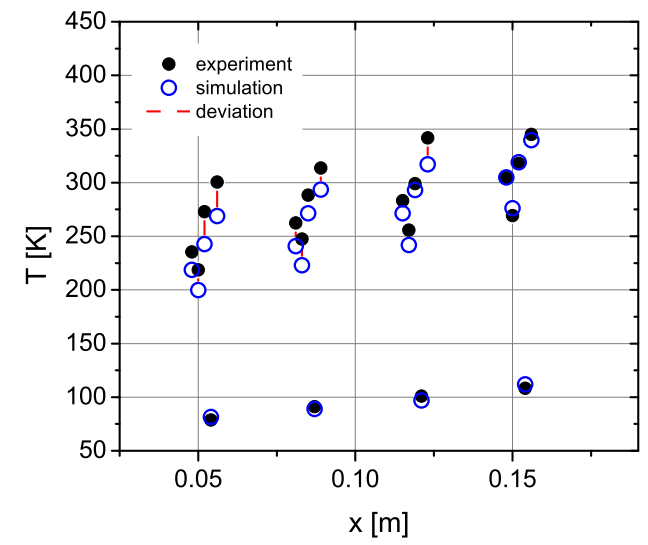

(d) S3 (AR:30)

Fig. 10. Direct comparison of simulated to measured structural temperature values (hydrogen).

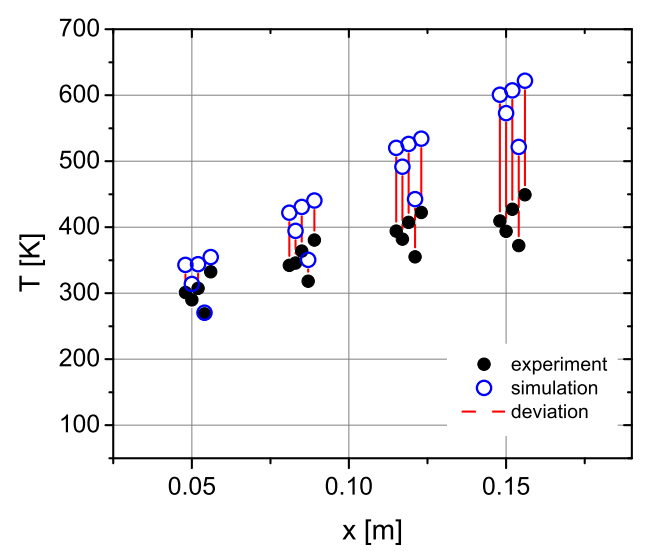

(a) $\mathrm{S} 1(\mathrm{AR}: 1,7)$

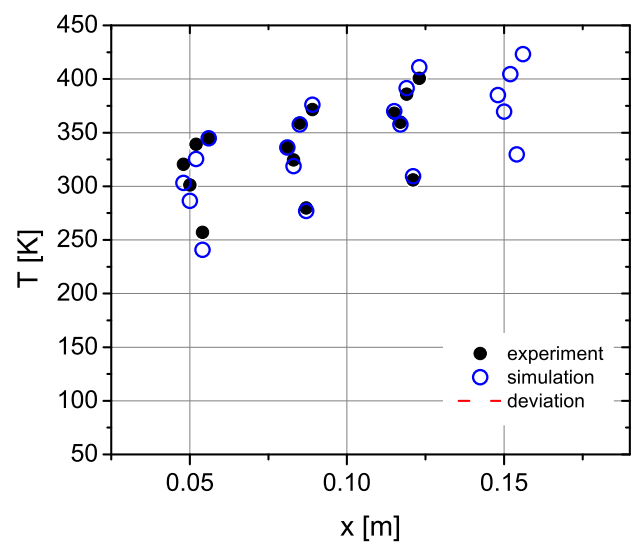

(b) S2 (AR:3,5)

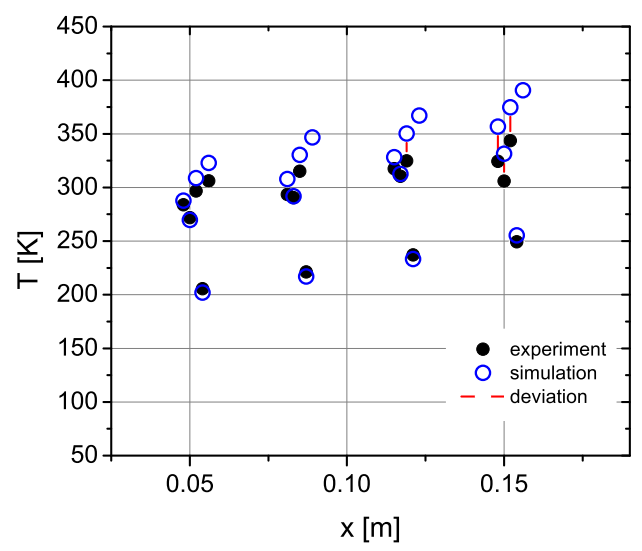

(c) $\mathrm{S} 4(\mathrm{AR}: 9,2)$

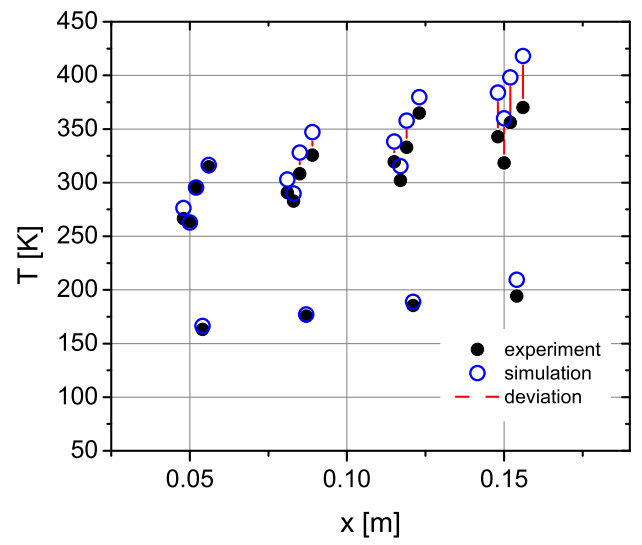

(d) S3 (AR:30)

Fig. 11. Direct comparison of simulated to measured structural temperature values (methane). 


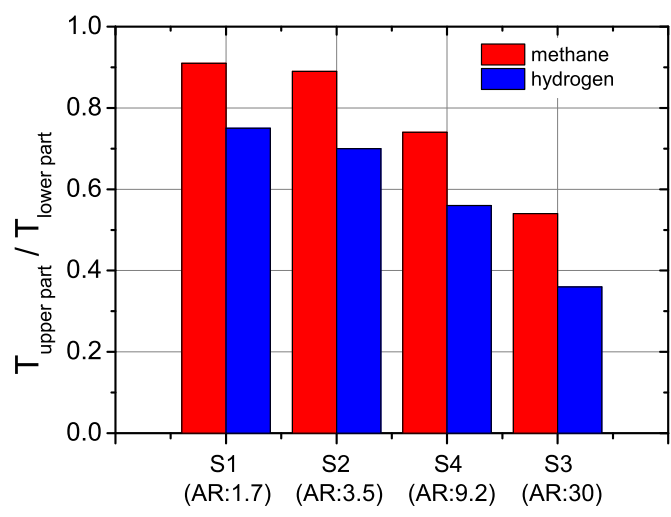

Fig. 12. Ratio of averaged temperature in the upper part and averaged temperature in the lower part of the cooling channels.

the hot gas side (the simulations underestimate these values) and for high aspect ratio channels S3 \& S4 cooled with methane for the temperatures close to the hot gas side wall.

Comparing the difference between temperatures close to the wall and temperatures far away from the hot gas side, the data show a larger difference for hydrogen and an increasing trend with increasing aspect ratio. This may limit thermal induced stresses inside the structure for cooling with methane. For cooling channel S3 the temperature values for the thermocouple that is farest away from the hot gass side wall is still close to the inlet temperature of the coolant. This indicates a strong thermal stratification of the cooling fluid that can be seen for both fluids.

\subsection{Thermal Stratification}

Due to a limited mixing in cooling channels with a very high aspect ratio the temprature in the lower part of the cooling channel is much higher than in the upper part. ${ }^{7}$ Figure 13 shows the temperature distribution at the outlet of the cooling channels for the testcases discussed above. For both cooling fluids, the temperature in the high aspect ratio channels (S4 \& S3) decrease with cooling channel height. For hydrogen this thermal stratification is more pronounced compared to methane. For hydrogen already the low aspect ratio channels (S1 \& S2) show this behavior and display a higher temperature at the bottom. In contrast to that, the temperature distribution for low aspect ratio channels cooled with methane, is even along the channel circumference but display a stratification towards the colder bulk flow. This is probably due to the high variations in fluid properties close to the critical point. Particularly the thermal conductivity exhibits a minimum value at the pseudocritical temperature that prevents thermal mixing.

To quantify these results the ratio between the average temperature in the upper $20 \%$ of the cooling channels to the lower $20 \%$ is calculated and displayed in fig. 12. This ratio decreases with increasing aspect ratio for both coolants. For hydrogen however the ratio is already low for the low aspect ratio cooling channels S1 \& S2, indicating already a certain stratification, whereas for methane the temperature distribution for these channels is nearly uniform. The higher the aspect ratio the stronger the thermal stratification gets. Especially for hydrogen cooling channel S3 (AR:30) exhibits a very strong stratification and accordingly both the highest and lowest temperature values in fig. 9 (a).

Thermal stratification can also be recognized when analyzing the heat flux around the circumference. Figure 14 shows

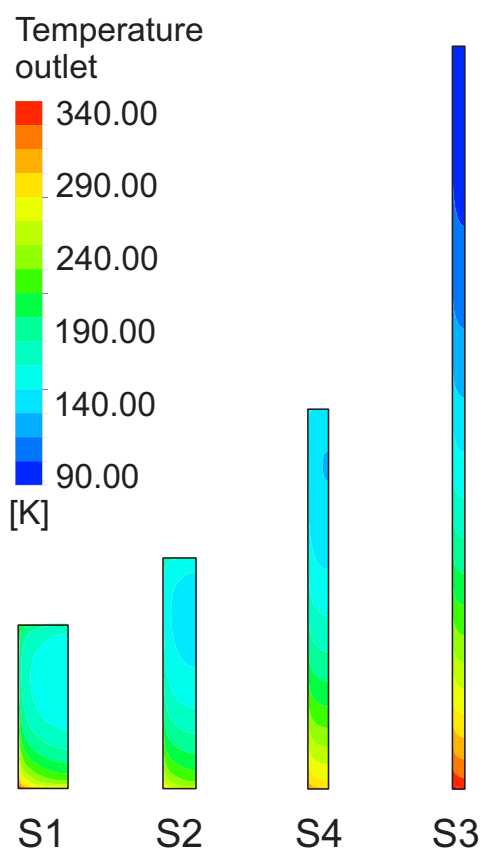

(a) Hydrogen

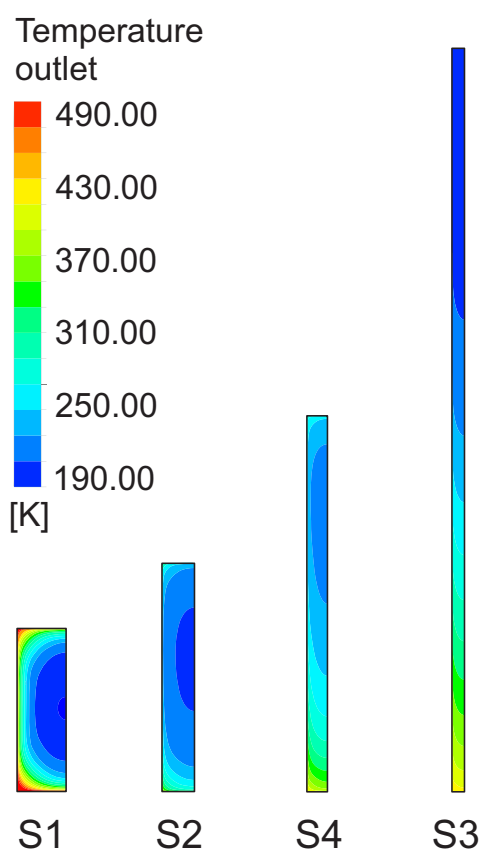

(b) Methane

Fig. 13. Temperature distribution at the outlet for all four sectors.

the heat flux around the circumference at measurement position 4 for each cooling channel and for both cooling fluids. For hydrogen, the heat flux decreases for the low and high aspect ratio channels along the channel height. Most of the heat is transferred in the lower part of the channel. For methane however, although a stratification is clearly visible, the heat flux is nearly constant along the circumference for the low and high aspect ratio channels. Thus the heat is transferred in both the lower and upper part of the channel. For cooling channel S3, a peak is discernable, close to the middle of that cooling channel. This effect is due to the peak of specific heat $c_{P}$ at the widom-line that leads lo a local improvement of the heat transfer. 


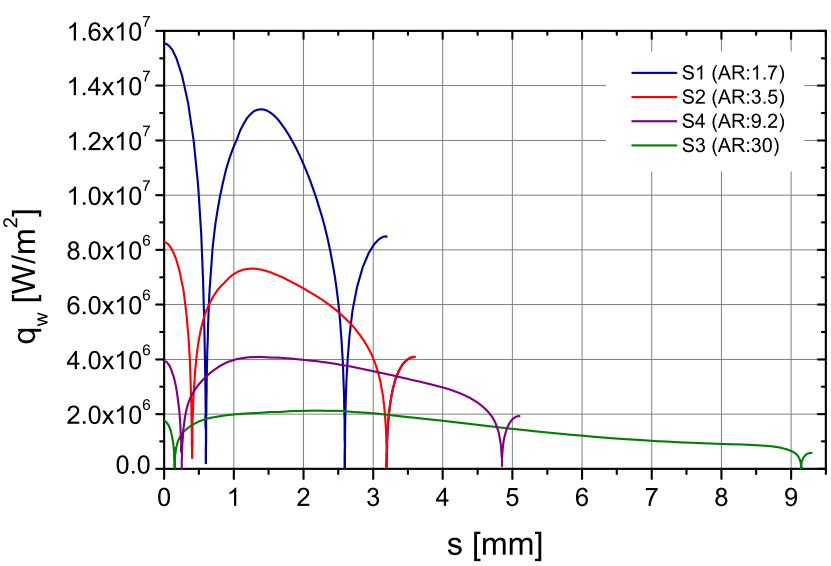

(a) Hydrogen

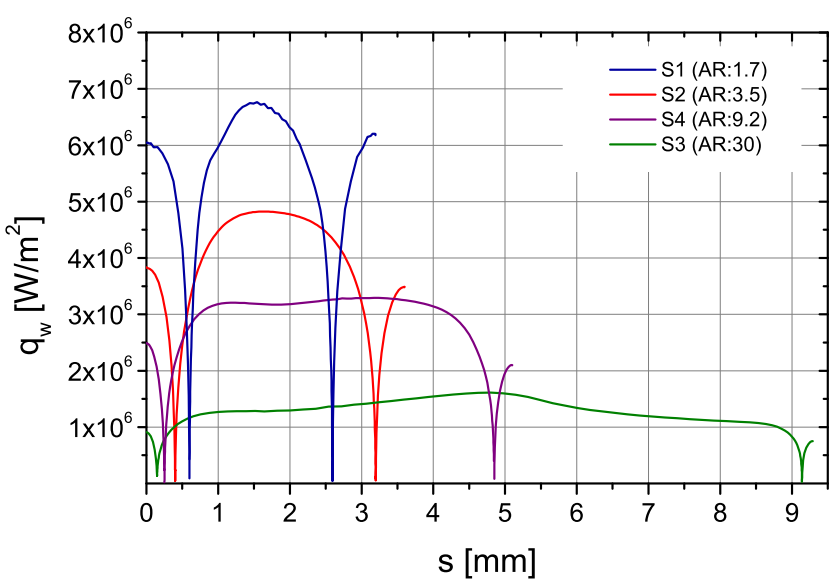

(b) Methane

Fig. 14. Heat flux around the circumference for all four sectors.

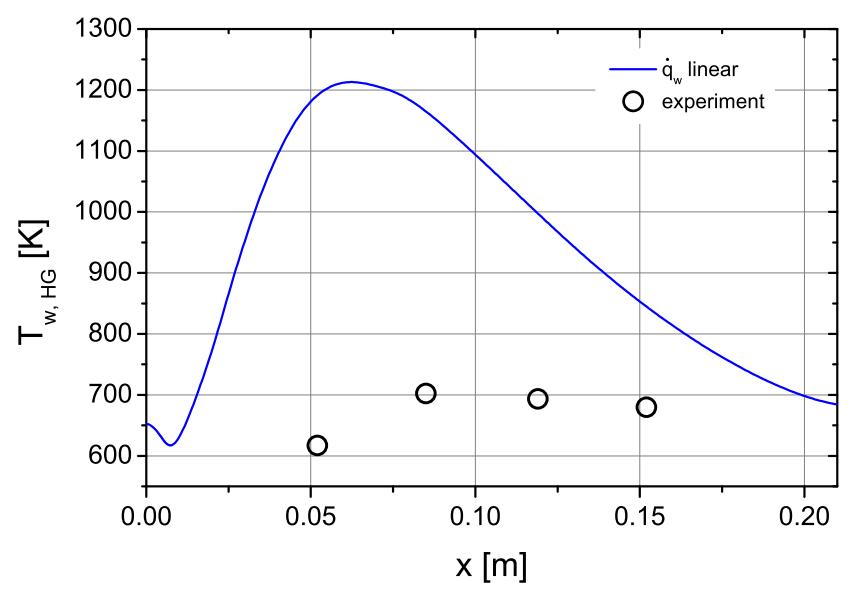

Fig. 15. Hot gas side wall temperature for a test case that exhibit HTD (S1 (AR:1.7)).

\subsection{Simulations close to the critical point}

Close to the critical point, the fluid properties change with small changes in temperature and pressure. Together with a high heat flux, this can lead to a separation of hot gas-like fluid at the wall and cold liquid-like fluid in the bulk flow. This leads to a locally decreased heat transfer coefficient. The phenomenon, called heat transfer deterioration is a major concern when methane is discussed as a coolant. This problem only occurs close to the critical point and is therefore not an issue for hydrogen.

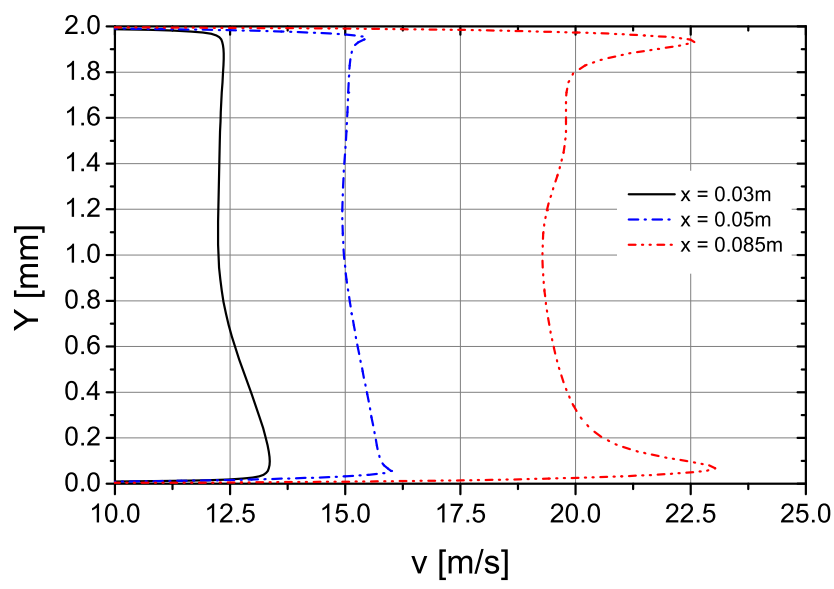

Fig. 16. Velocity at the symmetry line for a test case that exhibit HTD (S1 (AR:1.7)).

Figure 15 shows the wall temperature for $\mathrm{S} 1$ (AR:1.7) for a test case where heat transfer deterioration occurred and figure 16 shows the velocity profile at the symmetry line for this test case. Due to the lower density in the gas-like fluid at the wall, the fluid is accelerated and a characteristic M-shaped velocity profile is visible. Thus the simulations is capable to qualitatively describe the phenomenon that leads to the maximum in hot gas side wall temperature

However the simulations in that area show strong deviations to the experimental values. The turbulence model seems to underpredict the turbulent mixing between gas-like and liquid-like and accordingly overpredicts the hot gas side wall temperature. The simulation overpredicts not only the absolute values but also the onset of the maximum in axial direction. The temperature is higher and the temperature gradient is steeper compared to the experimental results.

Figure 17 shows the deviation $F$ between simulated hot gas side wall temperature $T_{w, H G, s i m}$ and measured $T_{w, H G \text {,exp }}$ hot gas

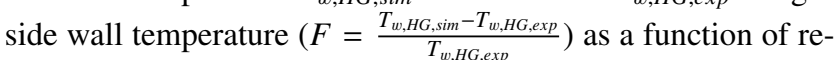
duced pressure and reduced temperature. The deviation is very strong close to the critical point, where HTD occurs, but decreases with increasing pressure. This effect may also be the reason for the high deviations for sector $\mathrm{S} 1$ in fig. 9(a).

Heat transfer deterioration primary occurs in cooling channels with a low aspect ratio. ${ }^{6)}$ In Sector 3 (AR: 30) no HTD was detected in the frame of these tests. Thus the deviation for the simulation of this sector is very low (see fig. 17(b)).

\section{Conclusion}

Experiments with a subscale combustion chamber at real conditions for hydrogen and methane as cooling fluids were performed that cover a wide range of thermodynamic conditions. These experiments were successful numerically rebuild. The agreement between experiment and simulation is very good. The comparison between hydrogen and methane show higher hot gas side wall temperatures and steeper gradients for methane. However the temperature decrease in radial direction as well as the difference between hot gas side wall temperature and structural temperature far away from the hot gas side is lower for the methane test case. Therefore thermal stresses are lower and the overall life time might be increased. 


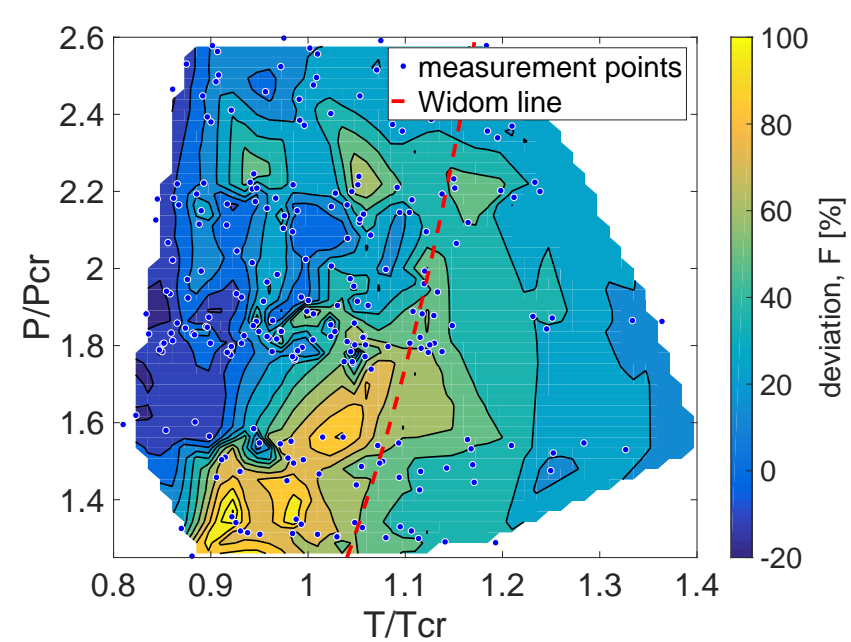

(a) Sector 1 (AR:1.7)

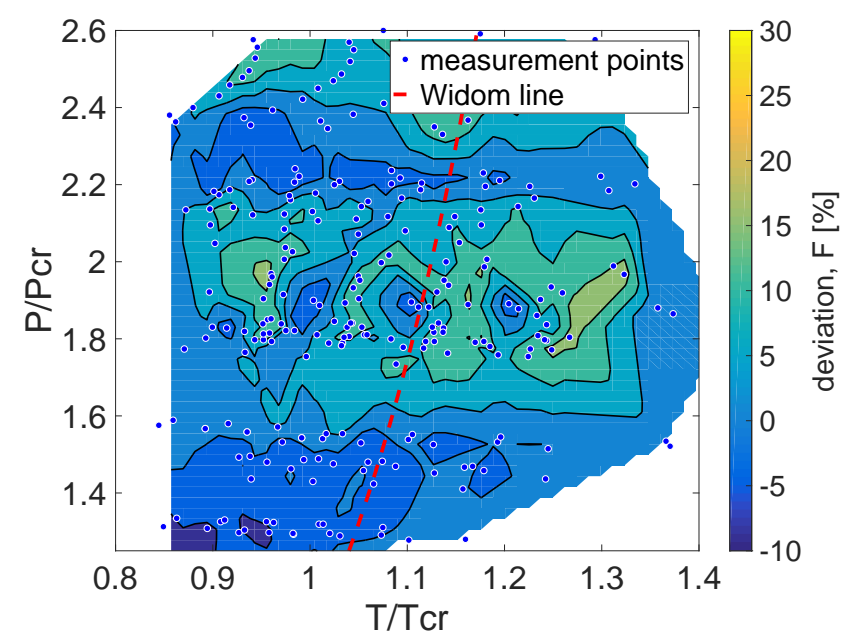

(b) Sector 3 (AR:30)

Fig. 17. Deviations for sector 1 and 3 for all test cases.

The thermal stratification occurs in cooling channels with a high aspect ratio for both fluids however it is more pronounced for hydrogen compared to methane. This also leads to a more homogeneous heat flux around the channels perimeter.

Heat transfer deterioration occurs in cooling fluids close to the critical point. The numerical simulations overpredict temperatures in this region and exhibit high deviations.

\section{References}

1) T. Adams, C. Grant, and H. Watson. A simple algorithm to relate measured surface roughness to equivalent sand-grain roughness. International Journal of Mechanical Engineering and Mechatronics Volume 1, Issue 1, Year 2012, 2012.

2) H. Asakawa, H. Nanri, K. Aoki, I. Kubota, H. Mori, Y. Ishikawa, K. Kimoto, S. Ishihara, and S. Ishizaki. The status of the research and development of lng rocket engines in japan. Springer Aerospace Technology, Chemical Rocket Propulsion: A Comprehesive Survey of Energetic Materials, 2017.

3) D. Banuti. Crossing the widom-line - supercritical pseudo-boiling. Journal of Supercritical Fluids(2015) 12 - 16, 2015.

4) M. Börner, C. Manfletti, J. Hardi, D. Suslov, G. Kroupa, and M. Oschwald. Laser ignition of a multi-injector lox/methane combustor. CEAS Space Journal (2018) 10:273-286, 2018.
5) J. Haemisch, D. Suslov, and M. Oschwald. Experimental analysis of heat transfer processes in cooling channels of a subscale combustion chamber at real thermal conditions for cryogenic hydrogen and methane. 6th Space Propulsion Conference, 14-18th May 2018, Sevilla, 2018.

6) J. Haemisch, D. Suslov, and M. Oschwald. Experimental study of methane heat transfer deterioration in a subscale combustion chamber. Journal of Propulsion and Power, 2019.

7) K. J. Kacynski. Thermal stratification potential in rocket engine coolant channels. Technical report, NASA, Lewis Research Center, Cleveland, Ohio, NASA-TM-4378, E-6135, NAS 1.15:4378, 1992.

8) T. Kato, D. Terakado, H. Nanri, T. Morito, I. Masuda, H. Asakawa, H. Sakaguchi, Y. Ishikawa, T. Inoue, S. Ishihara, and M. Sasaki. Subscale firing test for regenerative cooling lox/methane rocket engine. 7 th European Conference for Aeronautics and Space Science (EUCASS), 2017.

9) D. Kuhl, A. Holzer, and O. J. Haidn. Computational solution of the inverse heat conduction problem of rocket combustion chambers. 35th AIAA/ASME/SAE/ASEE Joint Propulsion Conference $\mathcal{E}$ Exhibit, June 20-23, 1999, Los Angeles, AIAA-99-2913, 1999.

10) J. W. Leachmann, R. Jacobsen, S. Penoncello, and E. Lemmon. Fundamental equations of state for parahydrogen, normal hydrogen, and orthohydrogen. J. Phys. Chem. Ref. Data, 38(3):721-748, 2009.

11) M. Leonardi, M. Pizzarelli, and F. Nasuti. Analysis of thermal stratification impact on the design of cooling channels for liquid rocket engines. International Journal of Heat and Mass Transfer 135 (2019) 811-821, 2019.

12) F. R. Menter. Two-equation eddy-viscosity turbulence models for enginnering applications. AIAA Journal, Vol 32, No8., 1994.

13) M. Oschwald, D. Suslov, J. Haemisch, O. Haidn, M. Celano, C. Kirchberger, N. Rackemann, A. Preuss, and D. Wiedmann. Measurement of heat transfer in liquid rocket combustors. High Pressure Flows for Propulsion Applications, AIAA Progress in Astronautics and Aeronautics Series, (not yet published).

14) M. Pizzarelli. The status of the research on the heat transfer deterioration in supercritical fluids: A review. International Communications in Heat and Mass Transfer, Volume 95, July 2018, Pages 132-138, 2018.

15) M. Pizzarelli, F. Nasuti, and M. Onofri. Analysis on the effect of channel aspect ratio on rocket thermal behavior. 48th AIAA/ASME/SAE/ASEE Joint Propulsion Conference $\mathcal{E}$ Exhibit 30 July - 1 August 2012, Atlanta, Georgia, 2012.

16) M. Pizzarelli, F. Nasuti, and M. Onofri. Trade-off analysis of highaspect-ratio-cooling-channels for rocket engines. International Journal of Heat and Fluid Flow, Volume 44, December 2013, Pages 458467, 2013.

17) R. J. Quentmeyer. Experimental fatigue life investigation of cylindrical thrust chambers. Technical report, NASA, Lewis Research Center, Cleveland, Ohio, 1977.

18) J. Sender, D. Suslov, J. Deeken, S. Gröning, and M. Oschwald. "142" technology demonstrator: Operational experience. Space Propulsion 2016, 2016.

19) U. Setzmann and W. Wagner. A new equation of state and tables of thermodynamic properties for methane covering the range from the melting line to $625 \mathrm{k}$ at pressures up to $1000 \mathrm{mpa}$. Journal of Physical and Chemical Reference Data, 20(6):1061-1151, 1991.

20) D. Suslov, A. Woschnak, D. Greuel, and M. Oschwald. Measurement techniques for investigation of heat transfer processes at european research and technology test facility p8. 1st EUCASS, 2005-07-04 2005-07-07,, 2005.

21) D. Suslov, A. Woschnak, J. Sender, M. Oschwald, and O. Haidn. Investigation of heat transfer processes in cooling channels of rocket engines at representative operating conditions. SPACE 2003, Moscow - Kaluga, Sept. 15-19 2003, Russian Federation, 2005.

22) A. Urbano and F. Nasuti. Onset of heat transfer deterioration in supercritical methane flow channels. Journal of Thermophysics and Heat Transfer Vol. 27, No. 2, April-June, 2013.

23) M. Wadel. Comparison of high aspect ratio cooling channel design for a rocket combustion chamber. Technical report, NASA, Lewis Research Center, Cleveland, Ohio 44135, 1997. 
24) M. F. Wadel and M. L. Meyer. Validation of high aspect ratio cooling in a $89 \mathrm{kn}$ thrust combustion chamber. Technical report, NASA, Lewis Research Center, Cleveland, Ohio, 44135, 1996.

25) A. Woschnak, D. Suslov, and M. Oschwald. Experimental and numer- ical investigations of thermal stratification effects. AIAA 2003-4615 39th Joint propulsion Conference, July, 20-23, Huntsville, Alabama, 2003. 